\title{
Focal therapy in urologic oncology: maximizing organ function and oncologic disease control
}

\author{
Jeffrey A. Cadeddu $\cdot$ Samir S. Taneja
}

Published online: 18 September 2010

(C) Springer-Verlag 2010

The widespread use of abdominal cross-sectional imaging has contributed to a rise in the diagnosis of small renal tumors. To minimize the risk of surgery-related chronic kidney disease, nephron-sparing surgery has now replaced the "gold standard" of radical nephrectomy in cases where complete removal of the tumor is possible without compromising the remnant portion of the kidney. While partial nephrectomy is associated with cancer control rates comparable to radical nephrectomy, both open and laparoscopic partial nephrectomy are associated with significant morbidity. Owing to the potential morbidity associated with extirpative surgery, investigation into alternative focal ablative therapies began in the late 1990s. These ablation technologies, primarily cryoablation and radiofrequency ablation, offer benefits over the extirpative approach including a lower complication rate, shorter convalescence, and absence of an ischemic insult. Collectively, these are significant considerations when taking into account that many small renal tumors are diagnosed in older patients with significant comorbidities. However, concerns regarding appropriate patient selection, oncologic efficacy, renal function preservation, and morbidity have limited the acceptance of renal focal therapy over the last decade.

J. A. Cadeddu

Department of Urology, University of Texas Southwestern Medical Center, Dallas, TX 75390-9110, USA

e-mail: jeffrey.cadeddu@utsouthwestern.edu

\section{S. S. Taneja $(\square)$}

Division of Urologic Oncology, Department of Urology, NYU Langone Medical Center, Urology Section, NYU Cancer Institute, Veterans Administration New York Harbor Healthcare System (Manhattan Campus), 150 East 32nd Street, Suite 200, New York, NY 10016, USA

e-mail: samir.taneja@nyumc.org
Fortunately, robust data are now available to the urologist regarding these parameters.

The contributors to this special topic provide a comprehensive review of appropriate patient selection criteria, imaging surveillance protocols and oncologic efficacy for both laparoscopic and percutaneous focal ablative techniques. After reading these articles, it is clear that oncologic efficacy and procedure morbidity are significantly influenced by two technical factors that are common denominators for focal prostate cancer therapy as well. First is tumor location as this influences both treatment success (i.e.: central/endophytic tumors have a moderately lower successful initial ablation rate) and risk of complications (i.e.: tumors near ureter risk stricture). Second is the appropriate imaging technique (US, CT) to monitor, assess, and follow ablation adequacy. After 10 years experience with focal therapy of kidney tumors, however, these parameters are now well defined, and we will see both cryoablation and radiofrequency ablation establish themselves as viable alternatives to partial nephrectomy.

While focal therapy for kidney cancer has been under evaluation for more than 20 years, and now may represent a standard of care for small early tumors, focal therapy for prostate cancer is an evolving frontier. A fundamental challenge of offering men with prostate cancer a focal therapy is the multifocal nature of the disease. Very few men with prostate cancer have unifocal disease, and it may be questionable whether those men would benefit from therapy at all. An emerging concept in focal therapy is that of the index lesion, or the focus of dominant disease in the gland. Early studies would suggest that this index lesion most often represents the greatest Gleason score, the most likely site of ECE, and, perhaps, the most likely source of metastatic progression. It remains to be seen whether control of the index lesion through focal ablation might 
sufficiently alter the natural history of the disease so as to avoid disease-related mortality.

Another fundamental hurdle in prostate cancer focal therapy is the limited tools available for disease localization. To date, the vast majority of focal therapy efforts have been guided by one of many saturation biopsy techniques. Improvements in magnetic resonance imaging, however, have opened the door to the possibility of image-guided focal therapy. Whether image-guided or biopsy-guided, paradigms for effective ablation, patient follow-up, and determination of long-term efficacy remain to be defined. In this issue, the invited articles provide a review of methods of candidate selection, novel methods of treatment, and known outcomes of therapy. It is our hope that these will serve both as an introduction to the concept of prostate cancer focal therapy and an impetus for the reader to evolve the rationale and practice of the field. 